\title{
ON YOUNG'S DEFINITION OF AN ALGEBRA*
}

BY L. E. BUSH

1. Introduction. The usual definition of linear algebra $\nmid$ presupposes a number field over which the algebra is taken. Some of the properties of the algebra are dependent upon the properties of this associated field, for instance, the property that if $e$ is an idempotent element, the subalgebra with basis $e$ is a field and therefore permits unique division by every non-zero element.

Young $\ddagger$ has given a definition of a general algebra and noted that a large part of the theory of linear algebras. is valid for it. This definition does not introduce the associated field. Young concludes his paper with "Precisely to what extent, if at all, the present formulation is more general than the current one in terms of a field is a problem of no little interest." He notes the fact that every Dickson algebra is a Young algebra. That the converse of this is not true, however, is shown by the simple example of a modular ring. In fact, a modular ring possesses an idempotent element which generates by addition alone the entire algebra, yet division is not unique. Ingraham $\S$ has noted this lack of restrictiveness of Young's definition, a lack of which, as I have been informed by Ingraham, Young had become cognizant.

The scalar multiplication in a Dickson algebra is not an operation within the algebra itself and it was with the view of eliminating this foreign operation that Young gave his definition. But, whether or not this operation is made a part of the definition of such an algebra, the possibility of defining an associated

* Presented to the Society, February 25, 1933.

† L. E. Dickson, Algebren und ihre Zahlentheorie, Zurich, 1927, pp. 23-24 and p. 32. A linear algebra as defined by Dickson will hereafter be referred to as a Dickson algebra.

$\ddagger$ J. W. Young, Annals of Mathematics, (2), vol. 29 (1927-28), pp. 54-60. A general algebra as defined by Young will hereafter be referred to as a Young algebra.

$\S$ M. H. Ingraham, this Bulletin, vol. 38 (1932), p. 100. 
field and a scalar multiplication is certainly a property of every Dickson algebra, and the lack of this property in the Young algebra gives it its greater generality.

Assuming that it would be desirable to give a definition for a Dickson algebra which makes use only of operations within the algebra itself, it is the purpose of this paper so to extend Young's definition by the addition of postulates as to make it equivalent to Dickson's definition. It will be pointed out that these postulates, as applied to a Dickson algebra, are equivalent to certain of Dickson's postulates on scalar multiplication.

2. Young's Definition. In brief, Young's definition is as follows. Let $\Im$ be a system of two or more elements and two operations, addition and multiplication, and let the elements of $₫$ form an abelian group, (5), under addition. A class [S్ $]$ of subgroups of $\$$ is called a partition of $\subseteq$ if every element $a$ (except the identity) of $(5)$ is in one and only one subgroup $\mathfrak{S}_{a}$ of [S్ $]$. The partition $[\mathfrak{S}]$ is called an $L$-partition if the subgroup $\left\{\mathfrak{S}_{1}, \mathfrak{S}_{2}, \cdots, \mathfrak{S}_{r}\right\}$ of $(5)$ generated by any finite number $r$ of subgroups $\mathfrak{S}$ is such that, if $\mathfrak{S}_{k}$ is any other $\mathfrak{S}$, then either $\left\{\mathfrak{S}_{1}, \mathfrak{S}_{2}, \cdots, \mathfrak{S}_{r}\right\}$ contains $\mathfrak{S}_{k}$ or it has no element in common with $\mathfrak{S}_{k}$ except the identity of $\$$

Let $[\mathfrak{S}]$ be an $L$-partition of $\mathfrak{S}$. Let $(a)$ denote that $\mathfrak{S}$ which contains the element $a$ and call it a simple linear set of $\subseteq$. By the product $(u)(v)$ of the simple linear sets $(u)$ and $(v)$ is understood the set of all elements of $\subseteq$ obtained by multiplying an element of $(u)$ by an element of $(v)$ in the order indicated. If for every choice of $u$ and $v,(u)(v)=(u v)$, where $(0)$ is the set consisting of the single element 0 (that is, the identity of $(\$),[\mathfrak{S}]$ is called an invariant L-pártition.

Denote by $(a, b, \cdots, p)$ the subgroup of (5) generated by $(a),(b), \cdots,(p)$ and call it a linear set of $\mathfrak{\subseteq}$. A finite set of elements $a_{1}, a_{2}, \cdots, a_{r}$ of $\mathfrak{S}$ is said to form a basis of order $r$ for $\mathfrak{S}$ if there exists an invariant $L$-partition of $\subseteq$, such that $\left(a_{1}\right)$, $\left(a_{2}\right), \cdots,\left(a_{r}\right)$ are all different and $\left(a_{1}, a_{2}, \cdots, a_{r}\right)=$ (5).

$A$ Young algebra $\mathfrak{A}$ of order $r$ ( $r$ finite) is a system consisting of a set $\subseteq$ of two or more elements, $a, b, \ldots$, and of two operations, addition $(+)$ and multiplication $(\cdot)$, and satisfying the following postulates. If $a, b$, and $c$ are any three elements of $\mathfrak{\subseteq}$,

A1. $a+b$ is a uniquely determined element of $\mathfrak{S}$. 
A2. $a+b=b+a$.

A3. $(a+b)+c=a+(b+c)$.

A4. There exists in $\subseteq$ an element 0 such that $a+0=a$.

A5. There exists in $\subseteq$ an element $-a$ such that $a+(-a)=0$.

From these postulates it follows that $\mathfrak{A}$ is an abelian group under addition and that the definitions in the first part of this section can therefore be applied to $\mathfrak{A}$.

M1. $a b$ is uniquely a determined element of $\mathfrak{S}$.

M2. $a(b+c)=a b+a c$.

M3. $(b+c) a=b a+c a$.

F. $\mathfrak{A}$ possesses a basis of order $r$ ( $r$ finite).

On the basis of these postulates Wedderburn's* calculus of complexes can be established and the first twenty-four theorems of his paper proved.

The definition of a Young algebra as given above is not exactly in the form in which Young stated it; however, only unessential changes have been made, particularly the explicit definition of the order of the algebra.

3. The Associated System. Let $\mathfrak{A}$ be a Young algebra. $\mathfrak{A}$ will be said to have isomorphic linear sets with respect to its basis if for every pair of elements $a$ and $b$ of $\mathfrak{A}$, neither of which is zero, there exists a simple isomorphism under addition between $(a)$ and $(b)$ such that $a$ as an element of $(a)$ corresponds to $b$ as an element of $(b)$.

Thus if $\mathfrak{A}$ has isomorphic linear sets there is an isomorphism between $(a)$ and $(b)$ in which any element $a^{\prime}$ of $(a)$ corresponds to any element $b^{\prime}$ of $(b)$. In particular, $(a)$ is isomorphic with itself in such a manner that an arbitrary element $a^{\prime}$ of $(a)$ corresponds to $a$. We shall introduce the abbreviation: $a^{\prime}$ in $(a) \sim b^{\prime}$ in (b), which is to be read $a^{\prime}$ as an element of (a) corresponds to $b^{\prime}$ as an element of $(b)$ in that isomorphism between $(a)$ and $(b)$ in which a corresponds to $b$.

If will be said to have equivalent linear sets with respect to its basis if

(1) it has isomorphic linear sets with respect to its basis;

(2) $a^{\prime}$ in $(a) \sim b^{\prime}$ in $(b), \quad c^{\prime}$ in $(c) \sim d^{\prime}$ in $(d), \quad(a c \neq 0, b d \neq 0)$, always implies $a^{\prime} c^{\prime}$ in $(a c) \sim b^{\prime} d^{\prime}$ in $(b d)$;

* J. H. M. Wedderburn, Proceedings of the London Mathematical Society, (2), vol. 6 (1908), pp. 79-81. Young, loc. cit., p. 60. 
(3) $a^{\prime \prime}$ in $\left(a^{\prime}\right) \sim b^{\prime}$ in $(b), \quad a^{\prime}$ in $(a) \sim c^{\prime}$ in $(c), \quad(b c \neq 0)$, always implies $a^{\prime \prime}$ in $(a) \sim b^{\prime} c^{\prime}$ in $(b c)$;

(4) $b$ in $\left(a_{1}+a_{2}\right) \sim a_{1}^{\prime}$ in $\left(a_{1}\right) \sim a_{2}^{\prime}$ in $\left(a_{2}\right)$

always implies $b=a_{1}^{\prime}+a_{2}^{\prime}$.

Let $\mathfrak{A}$ be a Young algebra with equivalent linear sets with respect to its basis and let $\mathfrak{A}^{2} \neq 0$. Let $\subseteq$ be a system consisting of a set of elements (which we shall denote by Greek letters) and of two operations, addition and multiplication. Let the elements of $\widetilde{S}$ be in bi-unique correspondence with the elements of the simple linear set $(a)$ of $\mathfrak{A}$, and let addition in $\mathfrak{S}$ be defined by postulating that $\subseteq$ be isomorphic with $(a)$ under addition. Since $\mathfrak{A}$ has equivalent linear sets, $\mathfrak{S}$ is isomorphic under addition with every simple linear set of $\mathfrak{A}$. We define the product $\alpha \beta$ of any two elements $\alpha$ and $\beta$ of $\subseteq$ as follows. Since $\mathfrak{A}^{2} \neq 0$, there exist in $\mathfrak{A}$ two elements $a$ and $b$ (which may be identical) such that $a b \neq 0$. Let $a^{\prime}$ in $(a) \sim \alpha$ in $\mathfrak{S}$ and $b^{\prime}$ in $(b) \sim \beta$ in $\mathfrak{S}$. The product $\alpha \beta$ is that element of $\subseteq$ which corresponds to the element $a^{\prime} b^{\prime}$ in $(a b)$. That $\alpha \beta$ is thus uniquely defined is a consequence of the equivalence of the linear sets, for if $c$ and $d$ are two other elements of $\mathfrak{U}$ such that $c d \neq 0$ and these elements are used instead of $a$ and $b$ to define $\alpha \beta$, we have by (2) that $c^{\prime} d^{\prime}$ in $(c d) \sim a^{\prime} b^{\prime}$ in $(a b)$ and are thus led to the same element $\alpha \beta$ of $\subseteq$. We shall call $\subseteq$ the system associated with the basis of $\mathfrak{A}$ from which these linear sets arise.

4. Completion of the Definition. In order to make Young's definition equivalent to that of Dickson we add to the postulates of $\$ 2$ two others.

Postulate E. Some basis of $\mathfrak{A}$ gives rise to equivalent linear sets. Such a basis will be called an E-basis of $\mathfrak{A}$.

FIEld Postulate. If $\mathfrak{U}^{2} \neq 0$, the system $\mathfrak{\subseteq}$ associated with some E-basis of $\mathfrak{A}$ is a field. If $\mathfrak{U}^{2}=0$, there exists a field simply isomorphic under addition with the linear sets of some E-basis of $\mathfrak{A}$.

The two following theorems show the equivalence of the definitions. In the proof of the first theorem it becomes evident that, as applied to a Dickson algebra, the postulates here added are equivalent to Dickson's postulates on scalar multiplication.*

THEOREM 1. Every Dickson algebra is a Young algebra which satisfies Postulate E and the Field Postulate.

* Dickson, loc. cit., p. 24, Postulates II and III. The subdivisions of these postulates are referred to below as II (1), etc. 
Let $\mathfrak{A}$ be a Dickson algebra of order $n$ over a field $\mathfrak{F}$ with the basis $e_{1}, e_{2}, \cdots, e_{n}$. Then $\mathfrak{A}$ is a Young algebra and its basis as given above is a basis in the sense of Young. We have to show that Postulate E and the Field Postulate are satisfied.

If $x=\sum_{i=1}^{n} x_{i} e_{i}$ is any element of $\mathfrak{A},(x)$ consists of all elements $\alpha x$ as $\alpha$ varies over $\mathfrak{F}$. Let $y=\sum_{i=1}^{n} y_{i} e_{i}$ be another element of $\mathfrak{A}$ ( $y$ may be identical with $x$ ). Consider the correspondence between $(x)$ and $(y)$ in which $\alpha x \sim \alpha y$. In this correspondence $x \sim y$. The correspondence is evidently an isomorphism under addition and therefore satisfies (1). Since $(\alpha x)(\beta y)=(\alpha \beta)(x y),{ }^{*}(2)$ is satisfied. Since $\alpha(\beta x)=(\alpha \beta) x, \dagger$ (3) is satisfied. Since $\alpha(x+y)$ $=\alpha x+\alpha y, \ddagger(4)$ is satisfied. Thus Postulate $\mathrm{E}$ is satisfied by $\mathfrak{A}$ and since $\mathfrak{F}$ is a field the Field Postulate is also satisfied.

It may be noted that although Dickson's postulates II (1) and III (1) are not explicitly made use of in the above proof, they are implicitly assumed in the two added postulates, that is, in the possibility of relating a field to the algebra in the particular manner assumed.

LеммA. If $a_{1}, a_{2}, \cdots, a_{n}$ are linearly independent $\$$ elements of a Young algebra which satisfies Postulate $\mathrm{E}$ and the Field Postulate and $a_{i}^{\prime}$ is in $\left(a_{i}\right)(i=1,2, \cdots, n)$ and $\sum_{i=1}^{n} a_{i}^{\prime}$ is in $\left(\sum_{i=1}^{n} a_{i}\right)$, then $a_{i}^{\prime}$ in $\left(a_{i}\right) \sim \sum_{i=1}^{n} a_{i}^{\prime}$ in $\left(\sum_{i=1}^{n} a_{i}\right)$.

For, let $c_{k}=\sum_{i=1}^{n} a_{i}-a_{k}$, so that $\sum_{i=1}^{n} a_{i}=a_{k}+c_{k}$, and let $b_{k}{ }^{\prime}$ and $c_{k}^{\prime}$ be such that $\sum_{i=1}^{n} a_{i}^{\prime}$ in $\left(a_{k}+c_{k}\right) \sim b_{k}^{\prime}$ in $\left(a_{k}\right) \sim c_{k}^{\prime}$ in $\left(c_{k}\right)$. Hence, by (4), $\sum_{i=1}^{n} a_{i}^{\prime}=b_{k}^{\prime}+c_{k}^{\prime}$. But $c_{k}^{\prime}$ is in $\left(c_{k}\right)$, hence in the linear set $\mathfrak{E}=\left(a_{1}, a_{2}, \cdots, a_{k-1}, a_{k+1}, \cdots, a_{n}\right), \|$ and $\sum_{i=1}^{n} a_{i}^{\prime}-a_{k}^{\prime}$ is for a similar reason in $\subseteq$. On the other hand $b_{k}^{\prime}$ and $a_{k}^{\prime}$ are in the linear set $\left(a_{k}\right)$, and $\left(a_{k}\right) \wedge \mathfrak{C}=0$. Therefore $b_{k}^{\prime}=a_{k}^{\prime} \uparrow$ and $a_{k}^{\prime}$ in $\left(a_{k}\right) \sim \sum_{i=1}^{n} a_{i}^{\prime}$ in $\left(\sum_{i=1}^{n} a_{i}\right)$.

Theorem 2. Every Young algebra which satisfies Postulate E and the Field Postulate is equivalent to a Dickson algebra.**

* See Dickson, II, (3).

$\dagger$ See Dickson, II, (2).

‡ See Dickson, III, (2).

$\S$ A set of elements is linearly independent in the sense of Young if no one of them belongs to the linear set generated by the others; Young, loc. cit., p. 57.

\| Young, loc. cit., p. 57.

II Young, loc. cit., p. 56.

** Equivalent to a Dickson algebra with respect to the operations within 
Let $\mathfrak{A}$ be a Young algebra of order $n$ having the basis $e_{1}$, $e_{2}, \cdots, e_{n}$ satisfying Postulate $\mathrm{E}$ and the Field Postulate. If $\mathfrak{A}^{2}=0$, there is a field $\mathfrak{F}$ isomorphic under addition with the linear sets $\left(e_{1}\right),\left(e_{2}\right), \cdots,\left(e_{n}\right)$. The zero Dickson algebra of order $n$ over $\mathfrak{F}$ is evidently equivalent to $\mathfrak{A}$.

Suppose $\mathfrak{A}^{2} \neq 0$, and let $\Im$ be thesystem associated with the given basis. Then $\Im$ is a field. Since $\mathfrak{A}=\left(e_{1}, e_{2}, \cdots, e_{n}\right), e_{i} e_{j}=\sum_{k=1}^{n} a_{i j k}$, where $a_{i j k}$ is in $\left(e_{k}\right)$. Let the elements of $\subseteq$ be denoted by Greek letters. Then $a_{i j k}$ in $\left(e_{k}\right) \sim \alpha_{i j k}$ in $\mathfrak{S}$. Consider the Dickson algebra of order $n$ over the field $\subseteq$ with the basis $e_{1}^{\prime}, e_{2}^{\prime}, \cdots, e_{n}^{\prime}$, where

$$
e_{i}^{\prime} e_{j}^{\prime}=\sum_{k=1}^{n} \alpha_{i j k} e_{k}^{\prime},
$$

the $\alpha_{i j k}$ being defined as above. If $x$ is any element of $\mathfrak{A}$, $x=\sum_{i=1}^{n} x_{i}$, where $x_{i}$ is in $\left(e_{i}\right)$. Let $x_{i}$ in $\left(e_{i}\right) \sim \beta_{i}$ in $\mathfrak{S}$. Then to the element $x$ of $\mathfrak{A}$ let correspond the element $x^{\prime}=\sum_{i=1}^{n} \beta_{i} e_{i}^{\prime}$ of the Dickson algebra $\mathfrak{A}^{\prime}$.

Let $x$ and $y$ be any two elements of $\mathfrak{A}$ and $x^{\prime}$ and $y^{\prime}$ the corresponding elements of $\mathfrak{A}$ '. Then

$$
x=\sum_{i=1}^{n} x_{i}, \quad y=\sum_{i=1}^{n} y_{i},
$$

where $x_{i}$ and $y_{i}$ are in $\left(e_{i}\right)$, and $x+y=\sum_{i=1}^{n}\left(x_{i}+y_{i}\right)$. Let $x_{i}$ in $\left(e_{i}\right) \sim \beta_{i}$ in $\mathfrak{S}$, and $y_{i}$ in $\left(e_{i}\right) \sim \gamma_{i}$ in $\subseteq$; then $x_{i}+y_{i}$ in $\left(e_{i}\right) \sim \beta_{i}+\gamma_{i}$ in $\mathfrak{S}$. The element $x+y$ in $\mathfrak{A} \sim$ the element

$$
\sum_{i=1}^{n}\left(\beta_{i}+\gamma_{i}\right) e_{i}^{\prime}=\sum_{i=1}^{n} \beta_{i} e_{i}^{\prime}+\sum_{i=1}^{n} \gamma_{i} e_{i}^{\prime}=x^{\prime}+y^{\prime}
$$

in $\mathfrak{A}^{\prime}$. Therefore $\mathfrak{U}$ and $\mathfrak{U}^{\prime}$ are isomorphic under addition.

Moreover,

$$
x y=\sum_{i, j=1}^{n} x_{i} y_{j}, \quad x^{\prime} y^{\prime}=\sum_{i, j=1}^{n} \beta_{i} \gamma_{j} e_{i}^{\prime} e_{j}^{\prime}, \quad x_{i} y_{j}=\sum_{k=1}^{n} b_{i j k},
$$

where $b_{i j k}$ is in $\left(e_{k}\right)$. Suppose $e_{i} e_{j} \neq 0$. Because of the invariance of the linear sets, $x_{i} y_{j}$ is in $\left(e_{i} e_{j}\right)$ and by the definition of multiplication in $\mathfrak{S}, x_{i} y_{j}$ in $\left(e_{i} e_{j}\right) \sim \beta_{i} \gamma_{j}$ in $\mathfrak{S}$. Let $a_{i j p_{s}},(s=1,2, \cdots, r)$,

the algebra, that is, a Dickson algebra can be constructed which is isomorphic with the given algebra under addition and multiplication. Although scalar multiplication is not defined for the given algebra this theorem shows the possibility of defining such an operation for it. 
be the set of all the $a_{i j k},(k=1,2, \cdots, n)$, which do not vanish. Then

$$
e_{i} e_{j}=\sum_{k=1}^{n} a_{i j k}=\sum_{s=1}^{n} a_{i j p_{s}} \neq 0
$$

and is contained in the linear set $\mathbb{E}=\left(e_{p_{1}}, e_{p_{2}}, \cdots, e_{p_{r}}\right)$. But $x_{i} y_{j}=\sum_{k=1}^{n} b_{i j k}$ is in $\left(e_{i} e_{j}\right)$ and therefore also in $\mathbb{C}^{*}{ }^{*}$ On the other hand, since $b_{i j k}$ is in $\left(e_{k}\right), \sum_{s=1}^{r} b_{i j p_{s}}$ is in $\mathbb{S}$ and it follows that

$$
\sum_{k=1}^{n} b_{i j k}-\sum_{s=1}^{r} b_{i j p_{s}}
$$

is in $\mathbb{S}$. But the latter is evidently in a linear set supplementary to $\mathfrak{S}$ in $\mathfrak{A}$ and is therefore zero. All the elements appearing in this latter expression which do not vanish are, however, linearly independent; while a sum of linearly independent elements can not vanish. $\dagger$ Hence $b_{i j k}=0, k \neq p_{s},(s=1,2, \cdots, r)$. Similarly, since $e_{i} e_{j}$ is in $\left(x_{i} y_{j}\right), b_{i j k}=0$ implies $a_{i j k}=0$. Such of the $a_{i j k}$ as are different from zero are linearly independent and by the Lemma $b_{i j k}$ in $\left(a_{i j k}\right) \sim x_{i} y_{j}$ in $\left(e_{i} e_{j}\right) \sim \beta_{i} \gamma_{j}$ in $\mathfrak{\subseteq}$. Because of the isomorphism of $\left(e_{k}\right)$ and $\Im$ under addition, $\alpha_{i j k}=0$ if and only if $a_{i j k}=0$. By (3), $b_{i j k}$ in $\left(e_{k}\right) \sim \beta_{i} \gamma_{j} \alpha_{i j k}$ in $\subseteq$, and $b_{i j k}$ as an element of $\mathfrak{A}$ therefore corresponds to $\beta_{i} \gamma_{j} \alpha_{i j k} e_{k}^{\prime}$ as an element of $\mathfrak{U}^{\prime}$. If $e_{i} e_{j}=0, a_{i j k}=0,(k=1,2, \cdots, n)$, and on account of the invariance of the linear sets $x_{i} y_{j}=0$, therefore $b_{i j k}=0$, $(k=1,2, \cdots, n)$. But $a_{i j k}=0$ implies $\alpha_{i j k}=0$, and $b_{i j k}=0$ as an element of $\mathfrak{A}$ still corresponds to $\beta_{i} \gamma_{j} \alpha_{i j k} e_{k}^{\prime}=0$ as an element of $\mathfrak{U}^{\prime}$. Because of the isomorphism of $\mathfrak{A}$ and $\mathfrak{U}^{\prime}$ under addition,

$$
\begin{aligned}
x y & =\sum_{i, j=1}^{n} x_{i} x_{j}=\sum_{i, j, k=1}^{n} b_{i j k} \text { in } A \sim \sum_{i, j, k=1}^{n} \beta_{i} \gamma_{j} \alpha_{i j k} e_{k}^{\prime} \\
& =\sum_{i, j=1}^{n} \beta_{i} \gamma_{j} e_{i}^{\prime} e_{j}^{\prime}=x^{\prime} y^{\prime} \text { in } A^{\prime} .
\end{aligned}
$$

Thus $\mathfrak{A}$ and $\mathfrak{A}^{\prime}$ are equivalent algebras.

The Ohio State University

* Young, loc. cit., p. 57.

† Young, loc. cit., p. 56. 